\title{
Early warning of coal and gas outburst based on abnormal gas emission
}

\author{
Ming Deng ${ }^{1, *}$ \\ ${ }^{1}$ College of Computer Science, Huainan Normal University, Huainan, Anhui 232038, China
}

\begin{abstract}
Taking the dynamic time series data of gas emission in mining face as the research object, the early warning model of coal and gas outburst was established based on single-time gas emission information function. Based on the data of 21118 heading face before outburst of Panyi Mine in Huainan, the single-time gas emission information function diagram was drawn, named as G-line diagram in short. The result showed that during normal production period, the entity of G-line diagram was small, which was close to a stable horizontal line. And before the outburst, the G-line diagram showed an upward trend. The negative and positive entity of G-line diagram became larger. At the same time, there were many times positive lines in the process of rising. According to the different shape, colour, length and other characteristics of G-line diagram, the change trend of coal body state in front of working face can be judged. Based on that, the outburst symptoms in the incubation stage of coal and gas outburst can be identified, and the early warning of outburst can be realized. It is of great significance to ensure the safety of mine production.
\end{abstract}

\section{Introduction}

The coal industry is a high-risk industry fighting against water, fire, gas, ground temperature, coal dust and roof disasters. Among all kinds of natural disasters in mines, coal and gas outburst is particularly serious. By the end of 2020, more than 20000 coal and gas outbursts have occurred in China's mines. On the one hand, a large number of coal and rock flow caused by outburst will destroy roadway support, ventilation facilities and production equipment; on the other hand, it will also bury people, suffocate people and even lead to gas explosion accidents, which seriously threaten the safety of workers. Coal and gas outburst prediction is one of the key links of comprehensive prevention and control system. Accurate and timely warning is of great significance to reduce the outstanding hazards and ensure the safety of human beings ${ }^{[1]}$.

The gas in coal seam exists in adsorption and dissociation state. The system is in dynamic balance when the external conditions remain unchanged. When affected by mining, the constraints of coal body is released quickly. A large number of adsorbed gas begins to desorb and releases elastic properties. As a result, the coal body is broken and moved. And together with free gas, the expansion gas flow is formed. An immense volume of deep gas gushes out, leading to the change of gas concentration in airflow. It is found that there is a certain relationship between the change of gas content and the ground acoustic signal. Before the occurrence of rock burst, the gas in the coal body intermittently gushes out. Whether the gas emission rises or fluctuates, it is the precursor information of outburst danger ${ }^{[2-5]}$. Based on the data sequence of gas emission from monitoring system, the precursor information of gas outburst can be mined. And the future state of coal body can be identified, too.

\section{Time series of gas emission in working face}

\subsection{Time series}

Time series are the monitoring data of the dynamic evolution process from a system, which are arranged in the same or different time intervals according to the sequence of their occurrence time. It contains the structural characteristics and development rules of related systems, and it has the characteristics of trend, analogy and correlation ${ }^{[6]}$.

(1) Trend

The present state is a continuation of the past state to some extent. The smaller the time interval, the closer the relationship between the two states. Studying the time series, the law or trend of system operation can be revealed, and the future behavior or state of the system is predicted.

\section{(2) Analogy}

Many things are similar in development and change. By estimating and correcting the deviation caused by different conditions, the performance process of the first developing thing can be analogized to the later one. It can realize the early warning of the future development

*Corresponding author: mdeng76@163.com 
of things. In the process of analogy, the relationship between the part and the whole should be worthy of attention.

(3) Correlation

The development and change of anything is not isolated, and it is determined in the process of interrelationship and influence with the development and change of other things. Causality is the most important and widely used form of expression. There is a similar functional relationship between cause and result. It provides the possibility for using causal relationship to establish model for coal and gas outburst early warning.

\subsection{Time series of gas emission}

Gas emission quantity of working face obtained by mine monitoring system is a group of dynamic data, arranged at a certain interval $(\Delta t)$, which is named gas emission concentration time series ( $G$ series). $G$ series is the corresponding output data of a coal-rock dynamical system, and it contains following information:

(1) It reflects the characteristic of coal-rock dynamical system under normal state (non-inoculation coal and gas outburst).

(2) It contains the outburst developing process information of coal-rock dynamical system.

$G$ series reflects the active result of all coal seam features which participate in the process of gas emission. It contains the dynamic information of coal and gas outburst, such as the number potential energy of coal mass ahead, the accumulated internal energy of coal gas, mechanical properties of coal mass, and permeability. By analyzing the gas emission concentration time series, the future state of characteristic of coal-rock dynamical system can be predicted ${ }^{[7]}$.

\section{Unit-time gas emission information function of $\mathbf{G}$ series}

\subsection{Definition}

Taking $\tau$ as the unit-time, the G Series is divided into several sub intervals, and the unit-time gas emission information function $F_{i}$ is constructed (G function for short). $F_{i}$ is as fellow:

$$
F_{i}=x_{i}\left(C_{i 0}, C_{i m i n}, C_{i m a x}, C_{i t}, \tau, C_{i m}, f_{i}, Q_{g i}\right)
$$

Where, $\tau$ refers to the unit-time, $C_{i 0}, C_{i t}$, and $C_{i m}$ refer to the initial value, final time value and average value of gas emission concentration in $\tau$ respectively, $C_{\text {imin }}$ and $C_{\text {imax }}$ refer to the minimum and maximum value of gas emission concentration in $\tau$ respectively, $C_{0}$ refers to reference value of variable value, $f_{i}$ refers to detection times in $\tau, Q_{g i}$ refers to gas emission $\left(Q_{g i}=C_{i} Q_{i}\right.$, where $Q_{i}$ refers to air volume, unit: $\left.\mathrm{m}^{3} / \mathrm{min}\right)$, respectively.

\subsection{Visualization processing}

The visualization of unit-time gas emission information function is based on drawing unit-time graph. The meaning of parameters of $\mathrm{G}$ function is shown in Figure 1.

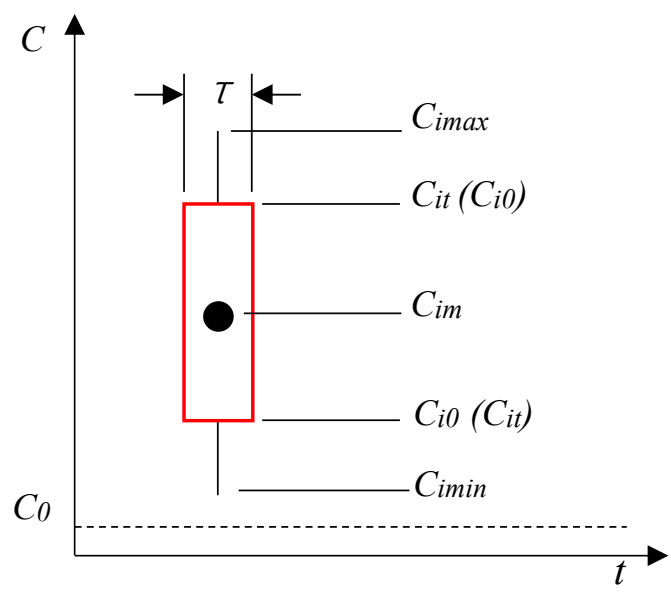

Fig. 1. The unit-time chart.

In the two-dimensional plane, with time as the horizontal axis and gas concentration as the vertical axis, each unit-time chart is drawn in turn, which is the unittime gas emission information function image (referred to as G-line diagram) (as shown in Figure 2). The unittime of G-line diagram can be divided into minutes, hours, days, etc.

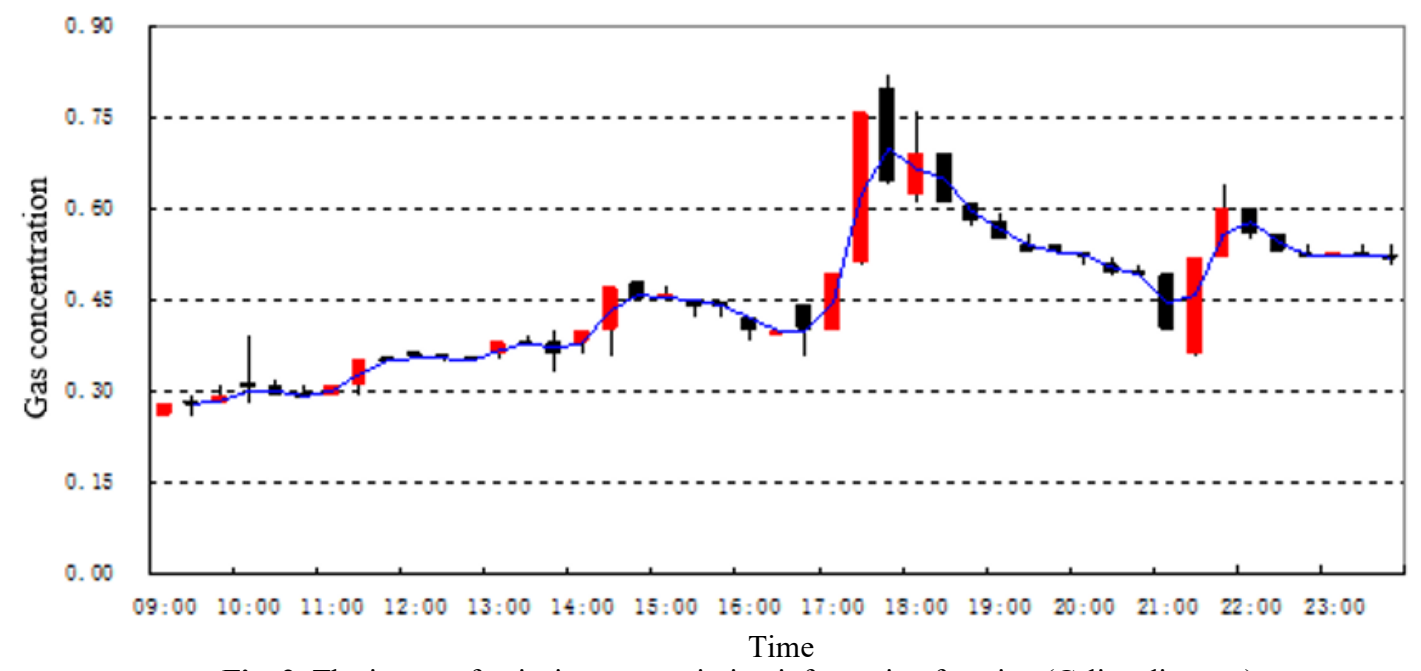

Fig. 2. The image of unit-time gas emission information function (G-line diagram) 
Unit-time gas emission information function is based on mathematical method. According to the type of Gline diagram, the equilibrium relationship between the outburst power (gas pressure, in-situ stress) and resistance (coal pillar binding force) can be analyzed, and the typical behavior of G-line diagram can also be concluded. Based on this, the state of gas bearing coal (rock) dynamic system can be predicted.

\section{Application of G-line diagram in early warning of coal and gas outburst}

Panyi Mine in Huainan Mining (Group) Co., Ltd. is a super large mine with an annual output of 3 million tons. The main coal seams are 13-1st coal, 11-2nd Coal and 8th coal, which are all high gas outburst coal seams. By the end of 2007, 25 times coal and gas outburst dynamic phenomena had occurred in the 13rd coal, 11th coal and 8th coal, including 8 times outbursts, with the maximum outburst intensity of 226 tons coal each time and 22366 $\mathrm{m}^{3}$ gas each time. The driving face of 21118 boundary inclined coal transportation roadway and return air combined roadway was driven in 8th coal seam, which was located between the coal outlet inclined roadway and the return air inclined roadway. At 9:00 on May 26, 2005 , the gas concentration after charging was $0.31 \%$, and the blast was conducted at 9:30. The gas concentration reached $0.4 \%$ in 30 minutes after the blast. At 12:08, coal and gas outburst occurred in the face. The coal and gas outburst extruded 320 tons coal and 6663 $\mathrm{m}^{3}$ gas.

The monitoring data before the outburst of the heading face in the return air combined roadway of the 21118 boundary coal conveying inclined roadway were obtained from the monitoring system, and the G-line diagram was drawn after preprocessing (as shown in Figure 3).

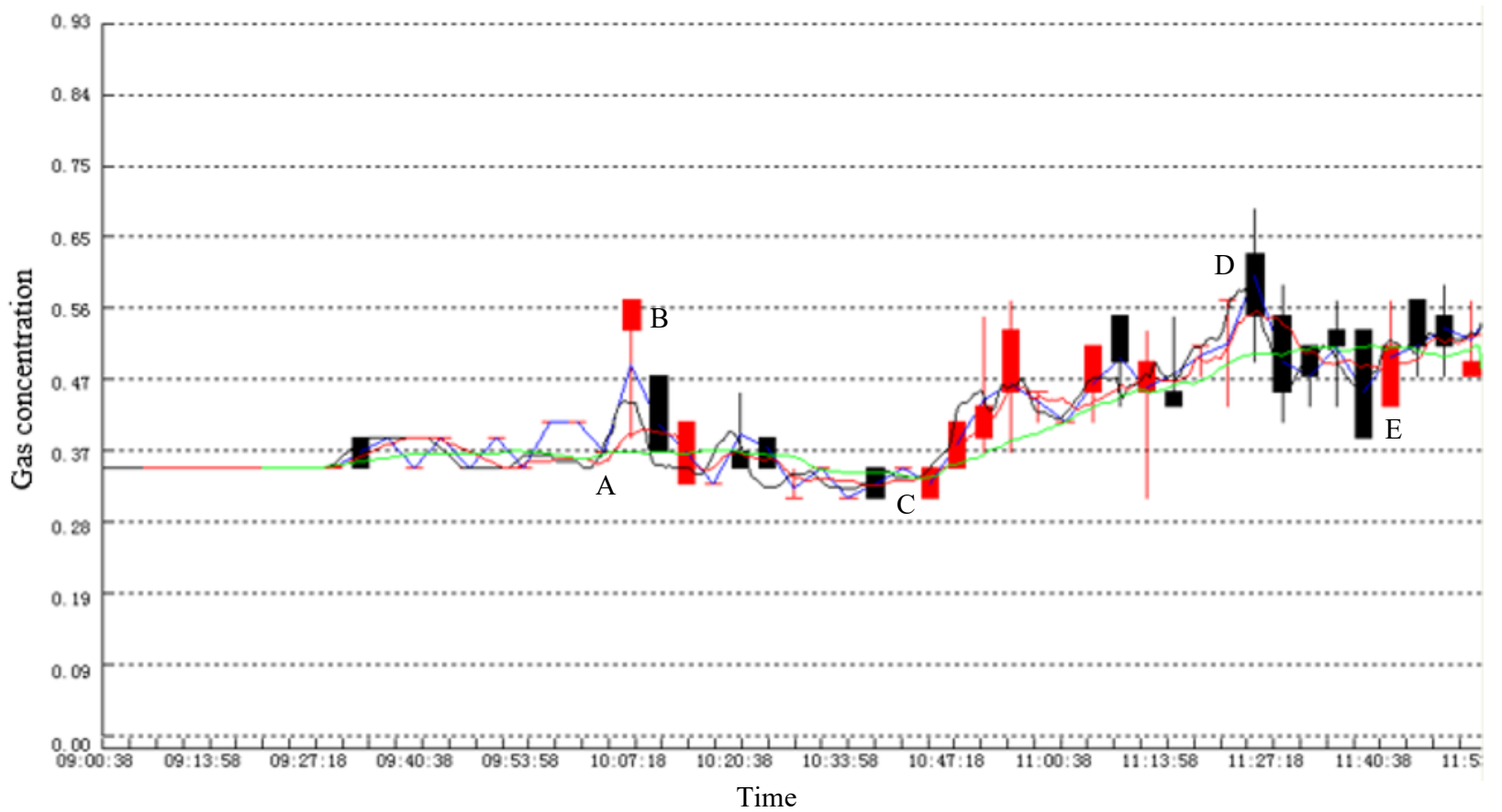

Fig. 3. G-line diagram of gas emission changes during embryonic stage of coal and gas outburst at 21118 driving face in Panyi Mine

Figure 3 shows that before 10:02:38 (mark A), the gas emission was stable, the $\mathrm{G}$ line alternately presented $\mathrm{T}$ and inverted $\mathrm{T}$ pattern, and the gas emission was stable. Then, $G$ line began to fluctuate up and down. At 10:05:38, the red entity with lower shadow line (mark B) appeared. The lower shadow line was longer than the red entity. The crustal stress and gas pressure increased, and the gas emission concentration began to rise. Then, the long red line (positive line) and long black line (negative line) alternated with each other, and the gas emission fluctuation increased. At 10: 43:08 (mark C), there were four continuous positive lines, and the gas emission concentration increased faster, which indicated that the coal body in front of the working face was more broken, the internal cracks of the coal body expanded, and the dynamic phenomenon gradually appeared. Then there was a descending process after 11:23:50 (mark D). A big positive line with shadow line suddenly appeared at 11:40:40 (mark E), and the red body was longer than the shadow line. This indicated that the coal and rock mass in front of the excavation was bound by the surrounding rock when the fractures were fully developed. In addition, the increase of gas emission concentration slowed down. However, the gas emission was in an upward trend totality. It was predicted that the dynamic system of gas bearing coal was out of balance, and the coal and gas outburst wound happen soon.

\section{Conclusion}

Before the occurrence of coal and gas outburst, it needs to go through a process of energy accumulation to make it gradually develop into a fragile equilibrium state of critical damage or even overload. In this process, gas emission shows certain regularity. Based on the time series of gas emission, the unit-time information function is established. In addition, the abnormal characteristics 
of gas before outburst can be obtained by analyzing the type of G-line diagram.

(1) During the normal production period, there is a dynamic balance among the gas pressure, crustal stress and coal (rock) column binding force. The gas emission concentration is relatively stable. The G-line diagram shows small G-line, cross line and small entity. The fluctuation range of the G-line diagram is small, and there is no obvious change trend.

(2) In the stage of outburst incubation, the restriction of outburst start is released gradually. A large number of micro cracks or fissures are formed in the gas bearing coal body. The adsorbed gas begins to desorb and forms gas flow together with free gas. Coal body is crushed and thrown in high-pressure gas flow, resulting in continuous outburst. The G-line diagram shows an upward trend as a whole. The positive line and negative line become larger obviously, and the peaks appear many times. The diagram shows a continuous positive line, which indicates that outburst power surge and gas power phenomenon will happen soon.

\section{Acknowledgments}

This work is supported by the Research Start-up Funds of Huainan Normal University (Research on early warning of coal mine disaster based on Wireless Sensor Network).

\section{References}

1. Frid V I. (1997) Electromagnetic radiation method for rock and gas outburst forecast. Journal of Applied Geophysics, 38:97-104.

2. Z.H. Li, E.Y.Wang and X.Q. He. (2017) Study of distribution of electromagnetic radiation of coal or rock before drivage face. Journal of China University of Mining \& Technology, 36:142-147.

3. W.S. Su. (1995) Probing into the prediction of gas and coal outburst with dynamic index of gas emission. Coal Engineer, 5:2-7.

4. M. Deng, G.S. Zhang, Q.H. Chen. (2010) Forecast of coal and gas outburst based on time series of gas concentration. Journal of China Coal Society,2:260 263.

5. H. Eckel, H.I. Katenberg. (1990) Using ventilation monitoring technology to forecast coal and gas outburst . Coal Engineer, 4:51-56.

6. R.X. Qin. (2004) Research on real time early warning of gas and coal outburst based on monitoring system. Anhui University of Science and Technology, Anhui.

7. R.X. Qin, G.S. Zhang, Y.D. Yang. (2006) Predicting coal and gas outburst with monitored gas consistence. Journal of China Coal Society, 31:599602. 